\title{
Enhancing sensitivity of QCM for dengue type 1 virus detection using graphene-based polymer composites
}

\author{
Krongkaew Navakul $^{1} \cdot$ Chak Sangma $^{1} \cdot$ Pa-thai Yenchitsomanus ${ }^{2} \cdot$ Suticha Chunta $^{3} \cdot$ Peter A. Lieberzeit $^{4}$ (i)
}

Received: 3 February 2021 / Revised: 22 April 2021 / Accepted: 14 May 2021 / Published online: 6 June 2021

(C) The Author(s) 2021

\begin{abstract}
Graphene oxide-molecularly imprinted polymer composites (GO-MIP) have attracted significant attention as recognition materials in sensing due to their outstanding properties in terms of electrical and thermal conductivity, high mechanical modulus, and the comparably straightforward way to functionalize them. The aim of this study was to design a MIP-based sensor recognition material and enhance its sensitivity by blending it with GO for sensing a harmful dengue hemorrhagic fever pathogen, namely the dengue type 1 virus (DENV-1). Polymer composites comprising GO incorporated to an acrylamide (AAM)/methacrylic acid (MAA)/methyl methacrylate (MMA)/ $N$-vinylpyrrolidone (VP) copolymer were synthesized and compared to the "pure" MIP, i.e., the copolymer without GO. The pure polymer revealed a zeta potential of $+9.9 \pm 0.5 \mathrm{mV}$, whereas GO sheets prepared have a zeta potential of $-60.3 \pm 2.7 \mathrm{mV}$. This results in an overall zeta potential of $-11.2 \pm 0.2 \mathrm{mV}$ of the composite. Such polymer composites seem appropriate to bind the positively charged DENV-1 particle $(+42.2 \pm 2.8 \mathrm{mV})$. GO-MIP coated onto $10-\mathrm{MHz}$ quartz crystal microbalance (QCM) sensors indeed revealed two times sensitivity compared to sensors based on the pure MIP. Furthermore, GO-polymer composites revealed imprinting factors of up to 21, compared to 3 of the pure MIP. When plotting the sensor characteristic in a semilogarithmic way, the composite sensor shows the linear response to DENV-1 in the concentration range from $10^{0}$ to $10^{3} \mathrm{pfu} \mathrm{mL}^{-1}$. The corresponding limits of detection $(\mathrm{S} / N=3)$ and quantification $(\mathrm{S} / N=10)$ are 0.58 and 1.94 pfu $\mathrm{mL}^{-1}$, respectively. Furthermore, imprinted polymer composites selectively bind DENV-1 without significant interference: DENV-2, DENV-3, DENV-4, respectively, yield 13-16\% of DENV-1 signal. The sensor requires only about 15-20 min to obtain a result.
\end{abstract}

Keywords Molecularly imprinted polymers · Graphene oxide $\cdot$ Composites $\cdot$ Quartz crystal microbalance $\cdot$ Dengue type 1 virus

Published in the topical collection Analytical Applications of Biomimetic Recognition Elements with guest editors Maria C. Moreno-Bondi and Elena Benito-Peña.

Peter A. Lieberzeit

peter.lieberzeit@univie.ac.at

1 Department of Chemistry, Faculty of Science, Kasetsart University, Chatuchak, Bangkok 10900, Thailand

2 Division of Molecular Genetics, Department of Research and Development, Faculty of Medicine Siriraj Hospital, Mahidol University, Bangkok 10700, Thailand

3 Department of Clinical Chemistry, Faculty of Medical Technology, Prince of Songkla University, Hat Yai, Songkhla 90110, Thailand

4 Department of Physical Chemistry, Faculty for Chemistry, University of Vienna, Währingerstraße 42, 1090 Vienna, Austria

\section{Introduction}

Dengue virus (DENV) is the cause of dengue hemorrhagic fever (DHF). It comprises four different serotypes, namely DENV-1, DENV-2, DENV-3, and DENV-4. DHF is a reemerging public health problem for populations of tropical and sub-tropical regions, especially in the hyperepidemic region of Southeast Asia [1]. Although all four serotypes widely spread and circulate in most of this area, DENV-1 (36\%) is the most common serotype in Thailand, followed by DENV-3 (27\%), DENV-2 (23\%), and DENV-4 (14\%) [2]. A patient infected for the first time produces unique neutralizing antibodies against the respective particular serotype. Unfortunately, subsequent infection with another serotype may lead to antibody-dependent enhancement, producing non-neutralizing antibodies. Those promote virus entry into host cells, leading to more severe damage to internal organs [3]. Till now, no specific antiviral medication or vaccine exists 
for treating DHF and protecting patients against DENV, respectively [4]. Therefore, rapid detection and identification of DENV serotype infection is necessary for early diagnosis as well as for preventing severe damage and decreasing the risk of medical complications and death. Nowadays, rapid tests targeting dengue $\operatorname{IgG/IgM}$ and nonstructural proteins 1 (NS1) are utilized to detect DENV, but they offer only limited serotype classification [4]. For actually assessing the serotype, one therefore still needs genetic approaches, namely reverse transcriptase-polymerase chain reaction (RT-PCR). The method, however, does not allow for quantifying the virus load in the sample. For that purpose, one can use the standard plaque-forming unit (PFU) staining assay that requires cell cultivation [3]. Both methods are costly, laborious, and time-consuming. To tackle those issues, we herein present a rapid approach to both classify and directly quantify a DENV serotype, namely DENV-1 based on molecularly imprinted polymers (MIPs) and mass-sensitive detection. MIPs mimic the functions of natural receptors or antibodies by revealing selective properties via their shape, size, and chemical functionality [5]. MIP synthesis relies on the self-organization of functional monomers and cross-linkers around a template species. After polymerization and removing the template from the polymer, the matrix contains selective recognition sites complementary to the template. Those allow for selectively re-binding the respective templates, e.g., biomolecules [6-8]. Such biospecies are interesting target analytes for MIP-based sensing, because the polymers are usually much cheaper and more stable than recognition species from nature. Among various applications, surface MIPs for biospecies [9] have been synthesized successfully for wide a range of different viruses, such as the tobacco mosaic virus (TMV) [10] and influenza A subtypes (e.g., H5N1, H5N3, H1N1, H1N3, and H6N1) [11]. In addition, blending the polymer matrix with reinforcing materials, e.g., graphene oxide (GO), carbon nanotubes (CNTs), and metal nanoparticles, has led to high-performance composites. GO has received special attention in this regard, because among others, it is useful to enhance the thermal, mechanical, and electrical properties of the resulting composite [12]. Generally speaking, GO contains hydrophilic functional groups which can be functionalized in the polymer both covalently and non-covalently [13]. However, when preparing composites, one has to keep in mind that GO tends to aggregate and thus requires hydrophilic/hydrophobic groups present in the polymer to undergo strong polarpolar interactions [14]. They also impact on the system sometimes by their bulky size [15]. However, substantial research focusses on new strategies to modify surfacefunctionalized graphene oxide sheets for a range of applications [12, 14, 16].
For instance, Liu et al. published a polyethylene glycol functionalized nanographene oxide for delivery of waterinsoluble cancer drug (SN-38) [17]. Cai et al. synthesized poly(ethylene amine) functionalized $\mathrm{GO} /$ silver nanocomposite to increase the stability and decrease the cytotoxicity of silver nanoparticles [18]. Chang et al. combined poly(methacrylamide) (PMAAM)-based MIP with GO to achieve a selective element to detect 2,4-dichlorophenol [19]. In this work, we assess the sensing properties of GOMIP composite layers on quartz crystal microbalance (QCM) resonators to detect DENV-1. The DENV-1 serotype serves as a proof of principle, because it is widespread in Thailand.

\section{Materials and methods}

\section{Synthesis of graphene oxide}

GO synthesis followed Hummer's method [20]. Briefly, we first dissolved graphite flakes (mesh size 300) by stirring them in a solution containing concentrated sulfuric acid and phosphoric acid in a ratio of 9:1 $(v / v)$, followed by slowly adding potassium permanganate. The mixture was then added to $150 \mathrm{~mL}$ of $3.33 \%(v / v)$ aqueous $\mathrm{H}_{2} \mathrm{O}_{2}$ solution, which led to a bright yellow color. The product was filtered via $0.2 \mu \mathrm{m}$ Nylon membrane and washed with water before centrifugation at $5000 \mathrm{rpm}$ for $30 \mathrm{~min}$ to obtain GO sheets.

\section{Preparation of dengue virus}

The different dengue virus strains (DENV-1 to DENV-4) were cultured in C6/36 Aedes albopictus mosquito cells. All processes were carried out at biosafety laboratory level 2 under the standard safety protocol at the Department of Research and Development, Faculty of Medicine Siriraj Hospital, Mahidol University, as previously described [21]. The amount of each DENV serotype was assessed via a plaque-forming unit (PFU) staining assay using a monolayer of Vero cells. After incubating for 7 days, we stained the cell monolayer with $1 \%(v / v)$ crystal violet in $20 \%(v / v)$ ethanol to visualize circular plaques caused by DENV infection. The viruses were inactivated with $\beta$-propiolactone following a protocol of the Center for Disease Control and Prevention (CDC) before freezing them at $-70{ }^{\circ} \mathrm{C}$.

\section{Fabrication of QCM transducers}

Dual gold electrodes were generated on 10-MHz AT-cut quartz wafers (13.8 $\mathrm{mm}$ in diameter and $168 \mu \mathrm{m}$ in thickness; Great Microtama Industries, Surabaya, Indonesia) using a brilliant gold paste (Heraeus; 12\%) via screen printing [22]. Then, the quartz plates were baked in the oven at $400{ }^{\circ} \mathrm{C}$ for $4 \mathrm{~h}$. Fundamental resonance and damping of QCM were 
Table 1 Composition of polymer recipes for optimizing MIP

\begin{tabular}{|c|c|c|c|c|c|c|}
\hline \multirow[t]{3}{*}{ Polymer types } & \multicolumn{6}{|c|}{ Chemicals (mg) } \\
\hline & \multicolumn{4}{|c|}{ Functional monomers } & \multirow{2}{*}{$\begin{array}{l}\text { Cross- } \\
\text { linker } \\
\text { DHEBA }\end{array}$} & \multirow{2}{*}{$\begin{array}{l}\text { Initiato } \\
\text { AIBN }\end{array}$} \\
\hline & AAM & MAA & MMA & VP & & \\
\hline A & 15.3 & 11.0 & 6.5 & 6.7 & 47.0 & 1.5 \\
\hline B & 5.0 & 12.0 & 12.0 & 6.7 & 47.0 & 1.5 \\
\hline $\mathrm{C}$ & 5.0 & 20.0 & 0 & 6.7 & 47.0 & 1.5 \\
\hline
\end{tabular}

monitored via an Agilent 8712ET network analyzer. QCM transducers with less than $-5 \mathrm{~dB}$ damping were chosen for further use.

\section{Preparation of DENV-1 imprinted graphene oxide- polymer composites}

Previously published MIP for "negatively charged" influenza virus [11] (polymer A) served as the starting point for finding optimal MIP for DENV-1, because the envelopes of both viruses are made up of glycoproteins. However, both viruses present different surface charges. Therefore, polymer optimization included varying the amount of functional monomers, acrylamide (AAM), methacrylic acid (MAA), methyl methacrylate (MMA), and $N$-vinylpyrrolidone (VP) and keeping the amount of $N, N^{\prime}$-(1,2-dihydroxyethylene) bisacrylamide (DHEBA) cross-1inker and 2, $2^{\prime}-\mathrm{Azobis}(2$ methylpropionitrile) initiator constant at 47 and $1.5 \mathrm{mg}$, respectively, as shown in Table 1 . This system had also proven useful as the matrix for electrochemical sensing of the dengue virus on electrodes enhanced with GO (i.e., without imprinting) [23]. Polymers A, B, and C were synthesized by dissolving all chemicals in $300 \mu \mathrm{L}$ dimethyl sulfoxide (DMSO). The solution was pre-polymerized at $60{ }^{\circ} \mathrm{C}$ until just prior to reaching the gel point (approximately $30 \mathrm{~min}$ ). In parallel, template virus stamps were prepared by coating $5 \mu \mathrm{L}$ of DENV-1 standard corresponding to $1 \times 10^{5} \mathrm{pfu} \mathrm{mL}^{-1}$ onto a bare glass plate, followed by sedimentation at $4{ }^{\circ} \mathrm{C}$ for $20 \mathrm{~min}$.

Scheme 1 represents the general approach of GO-polymer composite layer fabrication: First, a pre-polymer solution was prepared as previously mentioned. Then, we mixed a colloidal suspension of GO platelets in $0.01 \mathrm{M}$ PBS, $\mathrm{pH}=7.40$ at $\mathrm{c}=$ $0.15 \mathrm{mg} \mathrm{mL}^{-1}$ to the desired pre-polymer matrix at a ratio of $3: 2(v / v)$. Those composite batches were spin-coated at $3000 \mathrm{rpm}$ for $2 \mathrm{~min}$ over both QCM electrodes. Instantly, the virus template stamp was pressed into the pre-polymer composite film above one of the electrodes to yield the GOMIP. The polymer on the untreated electrode leads to the socalled non-imprinted polymer (GO-NIP) for reference. Then, the polymer layer on QCM was completely polymerized at a $55^{\circ} \mathrm{C}$ oven for $18 \mathrm{~h}$. Finally, templates were removed by stirring in an aqueous solution containing 1 part $10 \%(\mathrm{v} / \mathrm{v})$ acetic acid and 1 part $0.1 \%(w / v)$ sodium dodecyl sulfate (SDS) solution, respectively, followed by deionized water (DW) for $30 \mathrm{~min}$ each. In a similar way, we prepared sensors containing "standard" MIP and NIP (without GO).

\section{Characterization of polymer composites}

Scanning electron microscopy (SEM) was carried out on a Quanta 450 FEI SEM with a voltage of $20.0 \mathrm{keV}$. All samples
Scheme 1 Schematic representation of GO-MIP and corresponding GO-NIP synthesis and their layer fabrication on QCM for detecting DENV-1

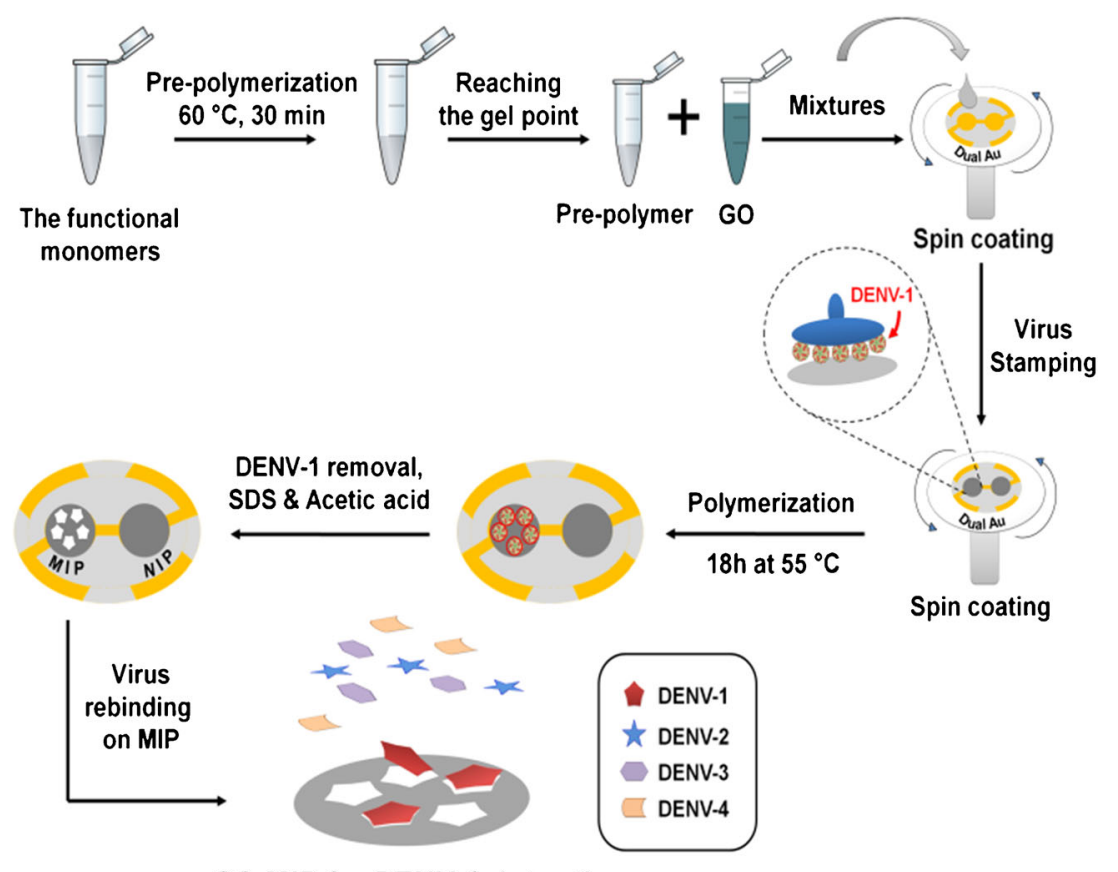

GO-MIP for DENV-1 detection 
were coated with a thin layer of Au by sputter-coating prior to SEM examination. Atomic force microscopy (AFM) served for characterizing morphologies of polymer surfaces on QCM using a Bruker Instruments NanoScope 8 in contact mode with silicon nitride cantilevers at a $1 \mathrm{~Hz}$ scan rate. To measure the zeta potentials of all material interfaces in $0.01 \mathrm{M}$ PBS, $\mathrm{pH}=7.40$ at $25^{\circ} \mathrm{C}$, we used a Malvern Zetasizer Nano-ZS90 instrument.

\section{QCM measurements}

Dual-electrode QCM were placed into a custom-made measuring cell, which connected to a home-built oscillator circuit linked to a frequency counter (Agilent 53131A) for a read-out. A custom-made LabView routine transferred measurement data to a computer by a GPIB/USB interface. All experiments took place in a stop-flow mode at room temperature $\left(25^{\circ} \mathrm{C}\right)$ to minimize sample volumes. For sensing, we mounted each QCM in a custom-made measuring cell, followed by obtaining equilibrium baseline signal through adding $180 \mu \mathrm{L}$ of $0.01 \mathrm{M}$ PBS, pH 7.40. Then, the sensor was exposed to $180 \mu \mathrm{L}$ of the respective DENV-1 standard in $0.01 \mathrm{M}$ PBS until reaching equilibrium. This was followed by washing with a mixed solution containing $10 \%(v / v)$ acetic ac$\mathrm{id} / 0.1 \%(w / v)$ SDS in a ratio of $1: 1$, followed by DW to regenerate MIP.

\section{Results and discussion}

\section{Optimizing polymer synthesis}

GO and DENV-1 in $0.01 \mathrm{M}$ PBS, $\mathrm{pH}=7.40$ have a net negative charge at $-60.3 \pm 2.7 \mathrm{mV}$ and a net positive charge at $+42.2 \pm 2.8 \mathrm{mV}$, respectively. Figure 1 collects SEM images of different surfaces, namely DENV-1 (Fig. 1A), GO (Fig. 1B), and GO with DENV-1 (Fig. 1C). Obviously, DENV-1 particles indeed show affinity toward the GO surface due to electrostatic

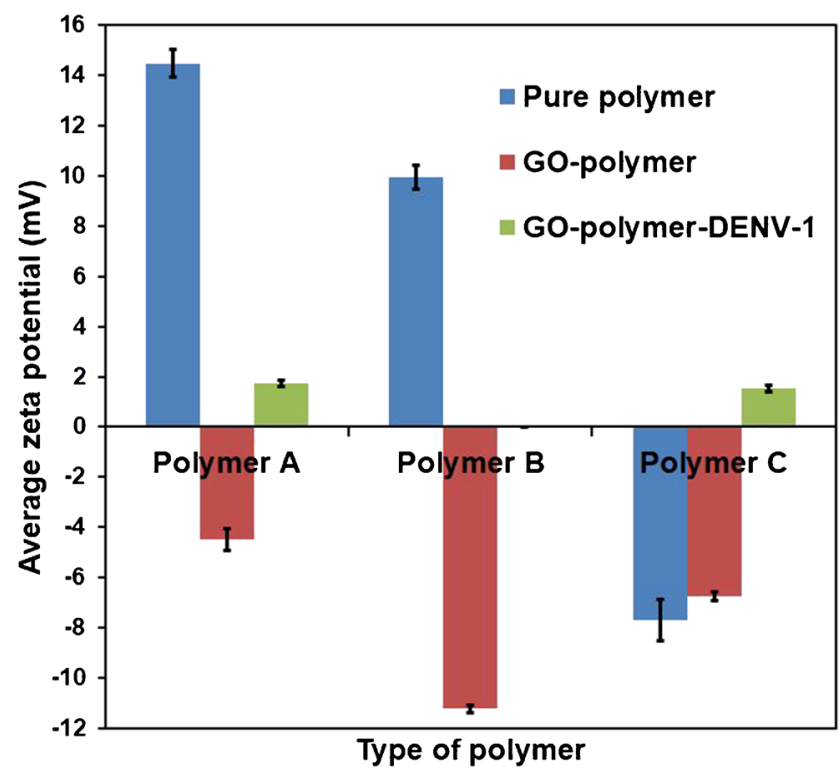

Fig. 2 Zeta potentials of copolymers $\mathbf{A}-\mathbf{C}$ (containing various ratios of AAM, MAA, MMA, VP), GO-polymer composites, and GO-polymerDENV-1

interactions. Of course, those are inherently non-selective, but affine. Therefore, it seems reasonable to optimize affinity between the receptor-i.e., the MIP — and its target analyte beyond "just" imprinting. Especially in aqueous solutions, electrostatic interactions play an important role in that regard. Figure 2 summarizes the zeta potentials of copolymers $\mathrm{A}-\mathrm{C}$, and their composites with $\mathrm{GO}$ and $\mathrm{GO} /$ DENV-1, respectively. DENV-1 on the one hand has a positive surface charge totaling $+42.2 \pm 2.8 \mathrm{mV}$ and on the other hand comprises a hydrophilic moiety comprising both negatively and positively charged side chains in the same way as other biomolecules. These observations have led to choosing AAM, MAA, MMA, and VP, as functional monomers, because they can provide a polymer with compositions of positive and negative charges. However, polymers $\mathrm{A}$ and $\mathrm{B}$ also reveal a positive surface charge at the respective values of $+14.5 \pm 0.5 \mathrm{mV}$ and $+9.9 \pm 0.5 \mathrm{mV}$, which makes them improbable candidates for binding a positively charged species such as DENV-1. In contrast
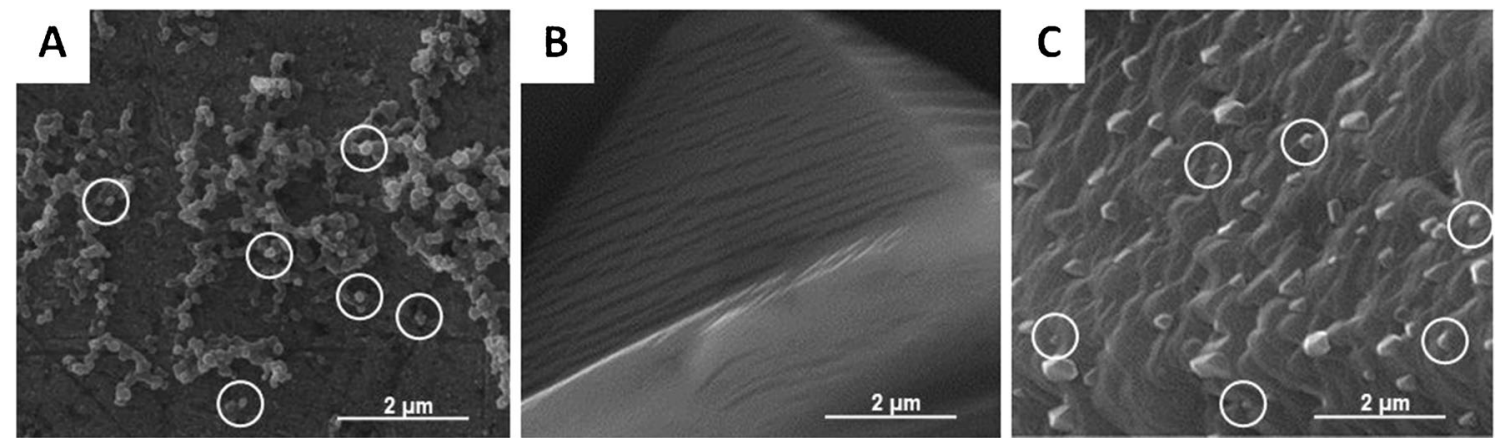

Fig. 1 SEM images of DENV-1 in 0.01 M PBS, pH=7.40 (A), GO in 0.01 M PBS, pH=7.40 (B), and DENV-1 on GO (C). (DENV-1 particles are indicated in white circles) 
to this, polymer $\mathrm{C}$ showed a negative surface charge at $7.7 \pm 0.8 \mathrm{mV}$ due to the increasing amount of negatively charged MAA. However, that value still seems rather low. On the other hand, GO sheets after synthesis led to a zeta potential of $-60.3 \pm 2.7 \mathrm{mV}$ due to the large number of carboxyl functionalities present on the surface. They indeed lead to a more negative surface charge of the respective GO-polymer composites, namely $-4.5 \pm 0.4 \mathrm{mV}$ for polymer $\mathrm{A},-11.2 \pm 0.2 \mathrm{mV}$ for polymer $\mathrm{B}$, and $-6.7 \pm$ 0.2 for polymer $\mathrm{C}$. After exposing all composites to a DENV-1 solution at a concentration of $10^{3} \mathrm{pfu} \mathrm{mL}^{-1}$, the zeta potentials of all polymers turned slightly positive again upon interaction: $+1.7 \pm 0.1 \mathrm{mV}$ for polymer $\mathrm{A}$, +
$0.006 \pm 0.003 \mathrm{mV}$ for polymer $\mathrm{B}$, and $+1.5 \pm 0.1 \mathrm{mV}$ for polymer $\mathrm{C}$. This indicates the inherent affinity of the GOpolymer to DENV-1, which is favorable for developing MIP. Overall, GO-polymer B reveals substantially higher negative surface potential than the other two composites which made it the best candidate for further experiments.

\section{Surface morphologies of classical polymers and composites}

Figure 3 displays AFM images of different surfaces, namely DENV-1-MIP before (Fig. 3A) and after (Fig. $3 \mathrm{~B}$ ) washing out the template, and GO-MIP before (Fig.
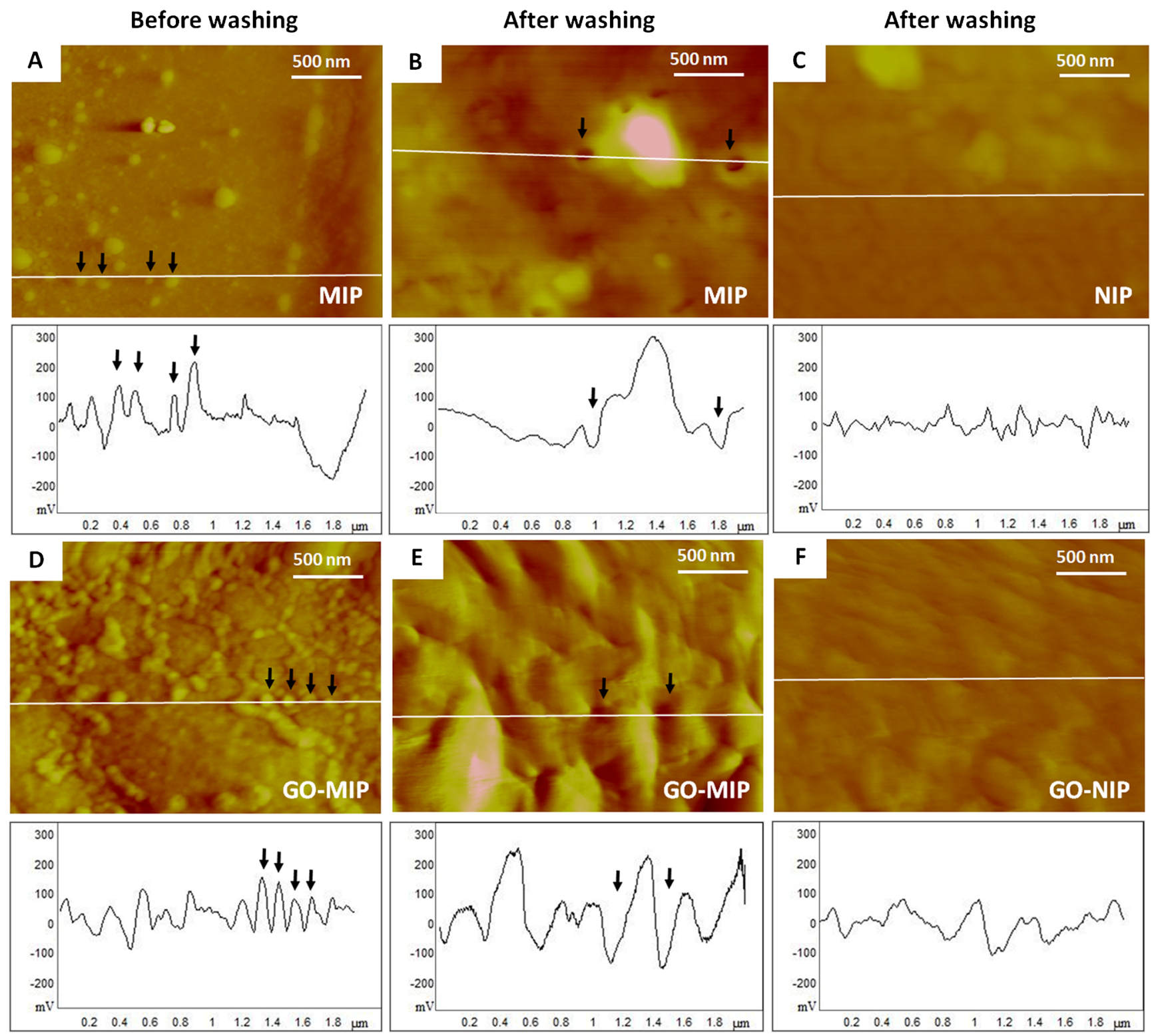

Fig. 3 AFM images and corresponding surface roughness profiles of polymers and $\mathrm{GO} /$ polymer composites (black linear graphs represent the selected cross-sections of the corresponding AFM image to show surface roughness. Some bound virus particles and imprinted cavities

are marked by dark arrows.): (A, D) MIP and GO-MIP before removing DENV-1 (B, E) MIP and GO-MIP after removing DENV-1 (C, F) NIP and GO-NIP 
3D) and after (Fig. 3E) the same steps, respectively, as well as their corresponding NIPs (Fig. 3C and F). Evidently, MIP surfaces before removing the virus are rough and reveal numerous independent roughly globular structures of DENV-1 with an average diameter of $96 \pm$ $8 \mathrm{~nm}$ on the surface. Deviations from the ideal shape may result from AFM measurements: in the case that tips are not ideally rotationally symmetric, one can observe such issues. In the same way, one can clearly see virus particles on the surface of the GO-MIP composite (with an average diameter of $93 \pm 7 \mathrm{~nm}$ ). After removing the template, the MIP and GO-MIP surfaces also show similar roughly globular cavities with the respective diameters of $94 \pm$ $5 \mathrm{~nm}$ and $88 \pm 8 \mathrm{~nm}$, corresponding to the size of DENV-1 particles. However, GO-MIP surfaces are rougher than those of MIP, because they contain GO sheets with sizes of 300-800 nm. Furthermore, neither NIP contains any of those features. These AFM images reveal that switching from the polymer to the composite does not change the shapes of the binding cavities revealed on the respective surface. They also clearly demonstrate that the three different polymer surfaces show drastically different morphologies.

\section{QCM sensing studies}

Figure 4 shows two sets of dual-electrode QCM responses: the first is the result of exposing a device carrying a DENV-1-MIP and NIP, respectively, when exposing them to a $10^{4} \mathrm{pfu} \mathrm{mL}^{-1}$ DENV-1 standard solution. This leads to frequency shifts of $595 \mathrm{~Hz}$ on the MIP side and $-197 \mathrm{~Hz}$ on the NIP side, corresponding to a $-399-\mathrm{Hz}$ mass effect, which provides evidence for successful imprinting. However, the imprinting factor (i.e., the signal ratio MIP/NIP) is 3 , which is rather low. The second sensor response is from a device coated with the two composites (GO-MIP and GO-NIP), respectively. In this case, exposing the device to the same standard solution of DENV-1 leads to signals of $-1690 \mathrm{~Hz}$ for GO-MIP and $-80 \mathrm{~Hz}$ for GO-NIP, hence $\mathrm{a}-1610-\mathrm{Hz}$ mass effect overall. The imprinting factor increases to 21 , which has a large impact on sensitivity. One can trace back such different response behaviors to surface charges: both the polymer $(+9.9 \pm 0.5 \mathrm{mV})$ and DENV-1 (+ $42.2 \pm 2.8 \mathrm{mV}$ ) have net positive surface charges, which obviously reduces the ability of the virus to efficiently bind to the MIP. The resulting sensor effect hence may be the result of comparably small enthalpic contributions combined with substantial entropy gain. Introducing GO into the polymer to yield GO-MIP, however, shifts the net surface charge of the thin film to negative values $(-11.2 \pm 0.2 \mathrm{mV})$ as previously mentioned. Hence, GO substantially increases the affinity of the respective MIPs and results in improved sensor responses. Moreover, the composites respond to the analytes faster than the pure polymers: their negative charges also increase the probability that a virus particle actually reaches a binding site on the MIP surface as a result of electrostatic attraction. It also leads to the shorter time for the signal to reach equilibrium, namely from roughly $5 \mathrm{~min}$ to the range of $1 \mathrm{~min}$. Both AFM images and QCM responses hence strongly support the claim of successful GO-MIP synthesis.

These promising results led us to investigate the sensor characteristics in a concentration range of $10^{-1}$ to $10^{4} \mathrm{pfu}$ $\mathrm{mL}^{-1} \mathrm{DENV}-1$. Figure 5A shows the frequency responses of GO-MIP and GO-NIP, respectively, as a function of time. Obviously, frequency shifts increase with increasing DENV1 concentrations with a dynamic range covering $10^{0}$ to $10^{4} \mathrm{pfu}$ $\mathrm{mL}^{-1}$. At the lowest concentration $\left(10^{-1} \mathrm{pfu} \mathrm{mL}^{-1}\right)$, the sensor response was too small $(\Delta \mathrm{f}=-26 \mathrm{~Hz}$; data not shown) to be statistically significant at a noise level of roughly $10 \mathrm{~Hz}$. Plotting the sensor characteristics in a semilogarithmic way reveals linear sensor characteristics of the GO-MIP sensor in the concentration range from 0 to 3 (i.e., $10^{0}-10^{3} \mathrm{pfu} \mathrm{mL}^{-1}$ ), as one can see in Fig. 5B. Data fit is excellent with a correlation coefficient of $\mathrm{R}^{2}=0.9981$. Limits of detection (LOD) and quantification (LOQ) were calculated on the basis of a $10-\mathrm{Hz}$ measurement noise, obtaining 0.58 and $1.94 \mathrm{pfu} \mathrm{mL}^{-1}$, respectively. Hence, the system is able to detect virus concentrations that correlate to early-stage infection with DENV-1. Compared to the MIP without graphene oxide, the composite is a factor of two more sensitive. Overall, the pure MIP led to $\mathrm{LOD}=0.60 \mathrm{pfu} \mathrm{mL}^{-1}$ and $\mathrm{LOQ}=2.0 \mathrm{pfu} \mathrm{mL}^{-1}$, which are higher than for the GO-MIP sensor. Therefore, rationally altering surface charge by synthesizing the composite pushes the sensitivity of the system toward the range required for

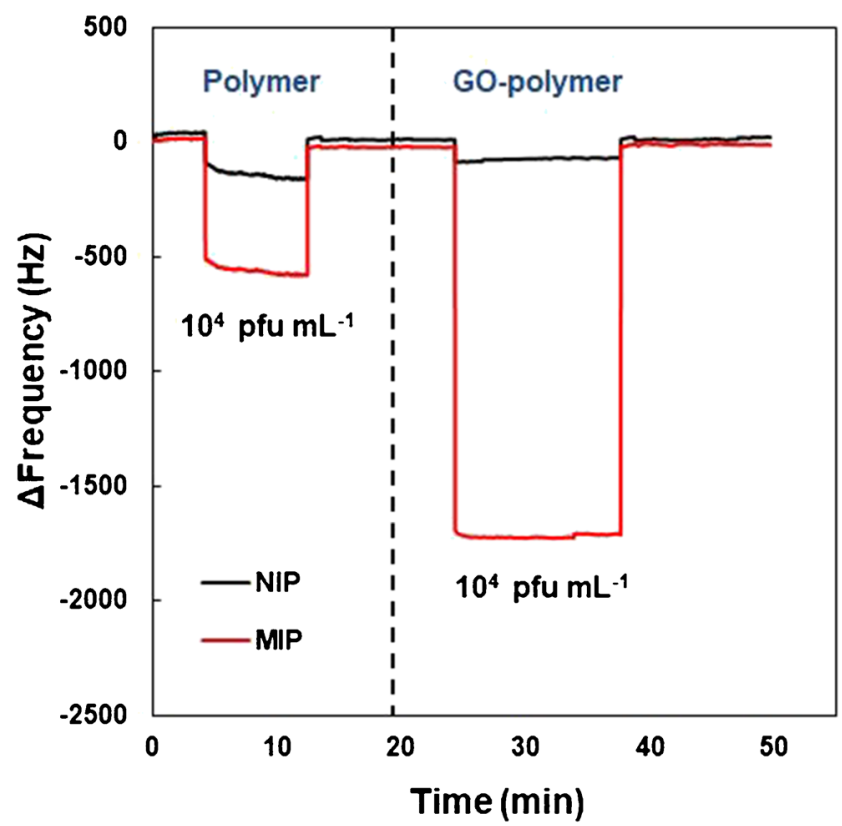

Fig. 4 QCM frequency responses of DENV-1-MIP/NIP and GO-DENV1 -MIP/NIP toward a standard DENV-1 in $0.01 \mathrm{M}$ PBS at $\mathrm{c}=10^{4} \mathrm{pfu}$ $\mathrm{mL}^{-1}$ 


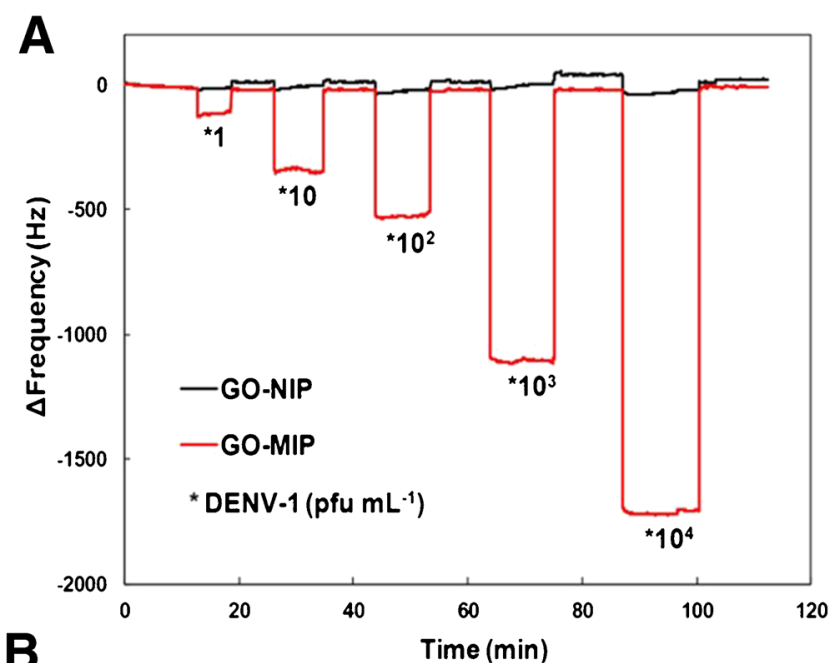

B

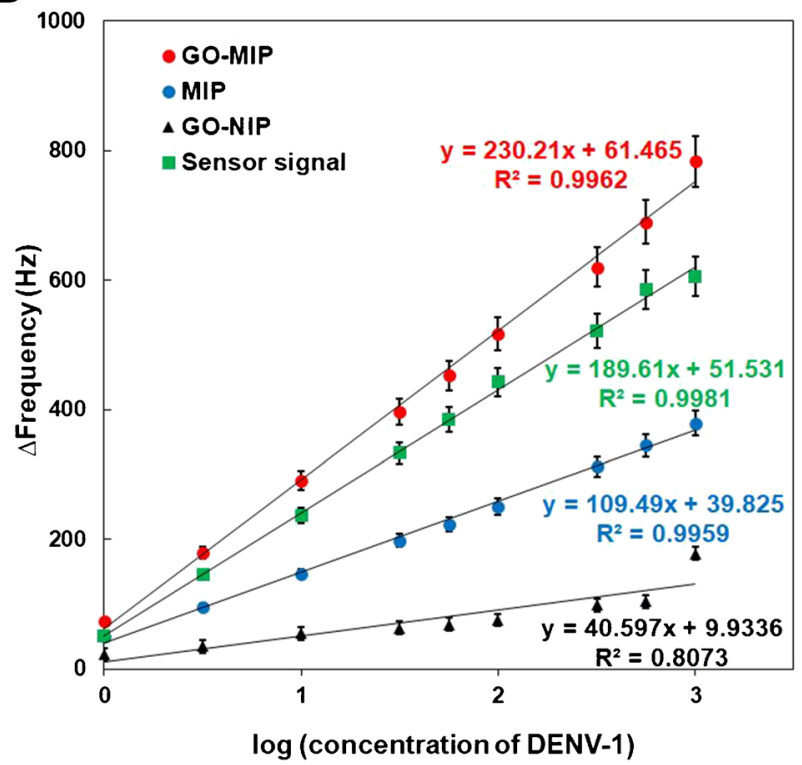

Fig. 5 GO-MIP/NIP sensor responses toward standard DENV-1 in $0.01 \mathrm{M}$ PBS with the range concentration of $10^{0}$ to $10^{4} \mathrm{pfu} \mathrm{mL}^{-1}$ (A); and linear response characteristic of GO-MIP, MIP, and GO-NIP toward standard DENV-1 in $0.01 \mathrm{M}$ PBS with the range concentration of $10^{\circ}$ to $10^{3} \mathrm{pfu} \mathrm{mL}^{-1}(\mathbf{B})$

actually applying those sensors in real-life settings, which is unusual for QCM-based sensing. However, imprinting also substantially contributes to sensitivity: the slope of the GONIP QCM sensor characteristic is $(\mathrm{k}=40.60)$ is roughly 2.5 times lower than that of the MIP and almost six times lower, than for the GO-MIP, respectively. Compared to the standard plaque assay that utilizes cell cultivation for virus quantification, this sensor strongly reduces analysis time from at least a 7-day incubation for plaque assay to within 15-20 min for sensor measurement.

\section{Selectivity}

As mentioned, there are four dengue virus serotypes (DENV1 to DENV-4) that differ by the specific antigens on the virus

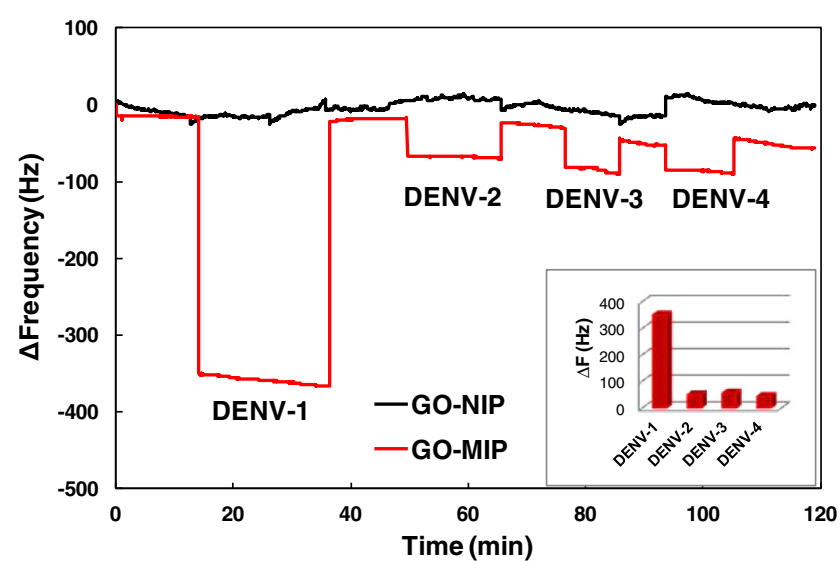

Fig. 6 GO-MIP/NIP sensor responses toward each serotype of DENV at a concentration of $50 \mathrm{pfu} \mathrm{mL}^{-1}$. The inset shows the relative effect compared to the respective DENV-1 signal

surface. Therefore, it is imperative to characterize the selectivity of MIPs and composites. Figure 6 summarizes the sensor responses of DENV1-GO-MIP and NIP, respectively, toward each serotype of DENV at a concentration of $50 \mathrm{pfu} \mathrm{mL}^{-1}$. The sensor responses for DENV-1, 2, 3, and 4, are -350,$51,-57$, and $-44 \mathrm{~Hz}$, respectively, corresponding to the relative effect of $100 \%, 15 \%, 16 \%$, and $13 \%$ of the DENV-1 signal. Such comparably low cross-reactivity between serotypes is even more surprising considering that they are 65 $70 \%$ homologous among each other [24]. Thus, the selectivity of MIP composites fits well with the functional differences between serotypes.

\section{Conclusions}

Graphene-based polymer composites allow for rationally altering the interaction properties of sensor layers by influencing surface charge. Though seemingly a "simple" step in sensor development, it shows substantial effects for detecting DENV-1 virions: GO-MIP composites lead to realistic limits of detection on QCM by increasing sensitivity by a factor of two compared to unmodified MIP. This study model of DENV-1 is promising for further studies on sensor arrays quantifying and identifying specific DENV serotypes in one measuring step of roughly $20 \mathrm{~min}$. This is much faster than any method based on cell culture or on nucleic acid amplification. In principle, the approach is expected to be feasible for mass-production, when using - harmless - pseudoviral particles for templating, i.e., virions lacking the viral genome.

Funding Open access funding provided by University of Vienna. This work is supported by the Department of Physical Chemistry, Faculty for Chemistry, University of Vienna, Austria, and the Faculty of Science, Kasetsart University, Thailand. 


\section{Declarations}

Conflict of interest The authors declare no competing interests.

Open Access This article is licensed under a Creative Commons Attribution 4.0 International License, which permits use, sharing, adaptation, distribution and reproduction in any medium or format, as long as you give appropriate credit to the original author(s) and the source, provide a link to the Creative Commons licence, and indicate if changes were made. The images or other third party material in this article are included in the article's Creative Commons licence, unless indicated otherwise in a credit line to the material. If material is not included in the article's Creative Commons licence and your intended use is not permitted by statutory regulation or exceeds the permitted use, you will need to obtain permission directly from the copyright holder. To view a copy of this licence, visit http://creativecommons.org/licenses/by/4.0/.

\section{References}

1. Sasmono RT, Taurel A-F, Prayitno A, Sitompul H, Yohan B, Hayati RF, et al. Dengue virus serotype distribution based on serological evidence in pediatric urban population in Indonesia. PLoS Negl Trop Dis. 2018;12(6):e0006616. https://doi.org/10.1371/ journal.pntd.0006616.

2. Fried JR, Gibbons RV, Kalayanarooj S, Thomas SJ, Srikiatkhachorn A, Yoon I-K, et al. Serotype-specific differences in the risk of dengue hemorrhagic fever: an analysis of data collected in Bangkok, Thailand from 1994 to 2006. PLoS Negl Trop Dis. 2010;4(3):e617-e. https://doi.org/10.1371/journal.pntd. 0000617.

3. Henchal EA, Putnak JR. The dengue viruses. Clin Microbiol Rev. 1990;3(4):376. https://doi.org/10.1128/CMR.3.4.376.

4. World Health O. Dengue guidelines for diagnosis, treatment, prevention and control: new edition. Geneva: World Health Organization; 2009.

5. Bossi A, Bonini F, Turner APF, Piletsky SA. Molecularly imprinted polymers for the recognition of proteins: the state of the art. Biosens Bioelectron. 2007;22(6):1131-7. https://doi.org/10. 1016/j.bios.2006.06.023.

6. Yan H, Row KH. Characteristic and synthetic approach of molecularly imprinted polymer. Int J Mol Sci. 2006;7(5):155-78.

7. Scheller FW, Zhang X, Yarman A, Wollenberger U, Gyurcsányi RE. Molecularly imprinted polymer-based electrochemical sensors for biopolymers. Curr Opin Electrochem. 2019;14:53-9. https:// doi.org/10.1016/j.coelec.2018.12.005.

8. Chunta S, Boonsriwong W, Wattanasin P, Naklua W, Lieberzeit PA. Direct assessment of very-low-density lipoprotein by mass sensitive sensor with molecularly imprinted polymers. Talanta. 2021;221:121549. https://doi.org/10.1016/j.talanta.2020.121549.

9. Eersels K, Lieberzeit P, Wagner P. A review on synthetic receptors for bioparticle detection created by surface-imprinting techniquesfrom principles to applications. ACS Sensors. 2016;1(10):1171-87. https://doi.org/10.1021/acssensors.6b00572.
10. Dickert FL, Hayden O, Bindeus R, Mann K-J, Blaas D, Waigmann E. Bioimprinted QCM sensors for virus detection - screening of plant sap. Anal Bioanal Chem. 2004;378(8):1929-34. https://doi. org/10.1007/s00216-004-2521-5.

11. Wangchareansak T, Thitithanyanont A, Chuakheaw D, Gleeson MP, Lieberzeit PA, Sangma C. Influenza A virus molecularly imprinted polymers and their application in virus sub-type classification. J Mater Chem B. 2013;1(16):2190-7. https://doi.org/10. 1039/C3TB00027C.

12. Potts JR, Dreyer DR, Bielawski CW, Ruoff RS. Graphene-based polymer nanocomposites. Polymer. 2011;52(1):5-25. https://doi. org/10.1016/j.polymer.2010.11.042.

13. Ding X, Chen X, Chen X, Zhao X, Li N. A QCM humidity sensor based on fullerene/graphene oxide nanocomposites with high quality factor. Sensors Actuators B Chem. 2018;266:534-42. https:// doi.org/10.1016/j.snb.2018.03.143.

14. Layek RK, Nandi AK. A review on synthesis and properties of polymer functionalized graphene. Polymer. 2013;54(19):5087103. https://doi.org/10.1016/j.polymer.2013.06.027.

15. Geim AK, Kim P. Carbon wonderland. Sci Am. 2008;298(4):90-7.

16. Neto AHC, Novoselov K. New directions in science and technology: two-dimensional crystals. Rep Prog Phys. 2011;74(8):082501. https://doi.org/10.1088/0034-4885/74/8/082501.

17. Liu Z, Robinson JT, Sun X, Dai H. PEGylated nanographene oxide for delivery of water-insoluble cancer drugs. J Am Chem Soc. 2008;130(33):10876-7. https://doi.org/10.1021/ja803688x.

18. Cai X, Lin M, Tan S, Mai W, Zhang Y, Liang Z, et al. The use of polyethyleneimine-modified reduced graphene oxide as a substrate for silver nanoparticles to produce a material with lower cytotoxicity and long-term antibacterial activity. Carbon. 2012;50(10):340715. https://doi.org/10.1016/j.carbon.2012.02.002.

19. Chang L, Wu S, Chen S, Li X. Preparation of graphene oxidemolecularly imprinted polymer composites via atom transfer radical polymerization. J Mater Sci. 2011;46(7):2024-9. https://doi.org/10. 1007/s10853-010-5033-z.

20. Hummers WS, Offeman RE. Preparation of graphitic oxide. J Am Chem Soc. 1958;80(6):1339. https://doi.org/10.1021/ja01539a017.

21. Punyadee N, Mairiang D, Thiemmeca S, Komoltri C, Pan-Ngum W, Chomanee N, et al. Microparticles provide a novel biomarker to predict severe clinical outcomes of dengue virus infection. J Virol. 2015;89(3):1587-607. https://doi.org/10.1128/JVI.02207-14.

22. Chunta S, Suedee R, Lieberzeit PA. Low-density lipoprotein sensor based on molecularly imprinted polymer. Anal Chem. 2016;88(2): 1419-25. https://doi.org/10.1021/acs.analchem.5b04091.

23. Navakul K, Warakulwit C, Yenchitsomanus PT, Panya A, Lieberzeit PA, Sangma C. A novel method for dengue virus detection and antibody screening using a graphene-polymer based electrochemical biosensor. Nanomed Nanotechnol. 2017;13(2):54957. https://doi.org/10.1016/j.nano.2016.08.009.

24. Azhar EI, Hashem AM, El-Kafrawy SA, Abol-Ela S, Abd-Alla AMM, Sohrab SS, et al. Complete genome sequencing and phylogenetic analysis of dengue type 1 virus isolated from Jeddah, Saudi Arabia. Virol J. 2015;12(1):1. https://doi.org/10.1186/s12985-0140235-7.

Publisher's note Springer Nature remains neutral with regard to jurisdictional claims in published maps and institutional affiliations. 